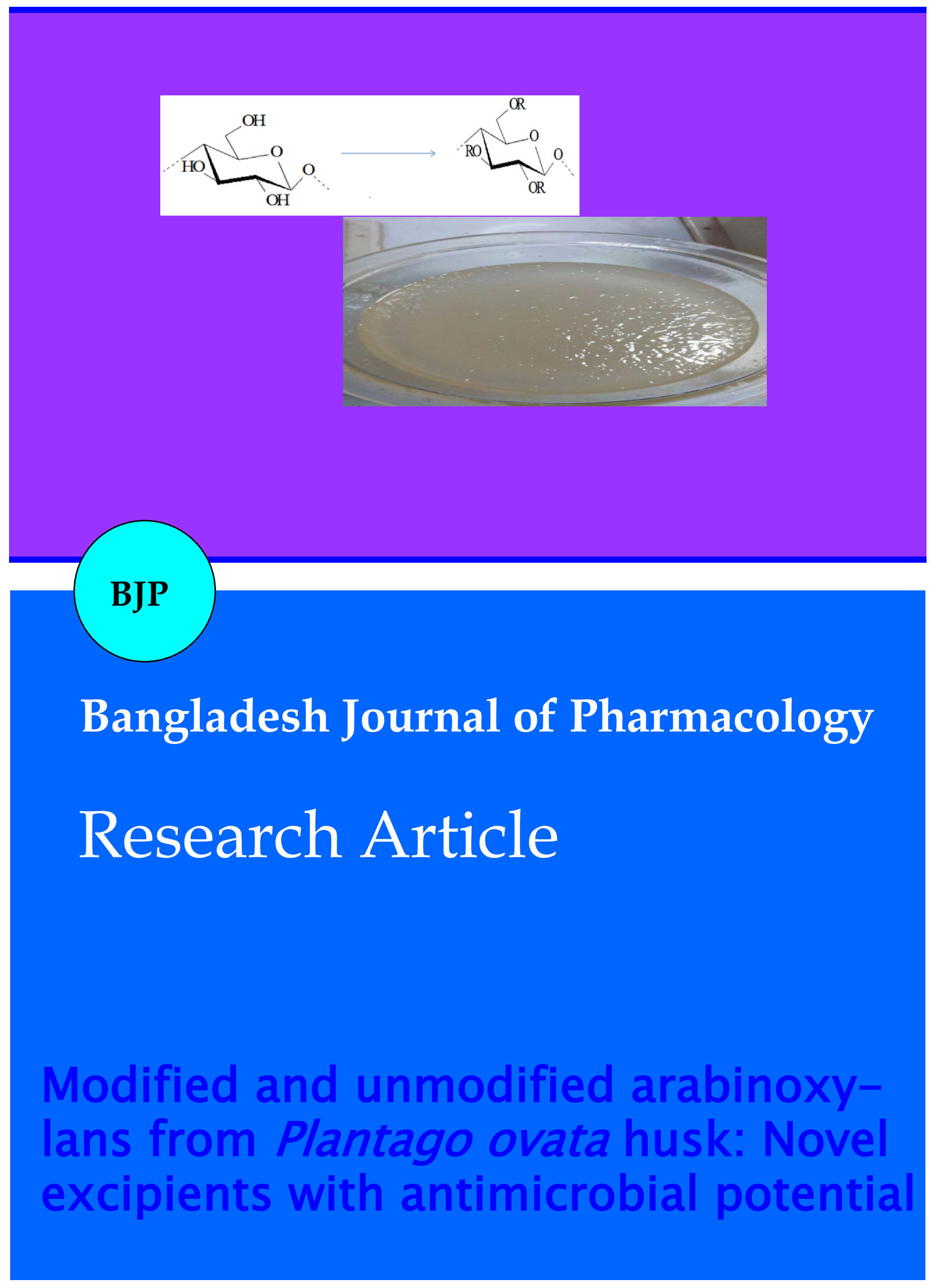




\title{
Modified and unmodified arabinoxylans from Plantago ovata husk: Novel excipients with antimicrobial potential
}

\author{
Alia Erum', Sajid Bashir' ${ }^{1}$ and Shazia Saghir ${ }^{2}$ \\ ${ }^{1}$ Faculty of Pharmacy, University of Sargodha, Sargodha, Pakistan; ${ }^{2}$ Department of Chemistry, Government Jinnah \\ Degree College for Women, Mozang, Lahore, Pakistan.
}

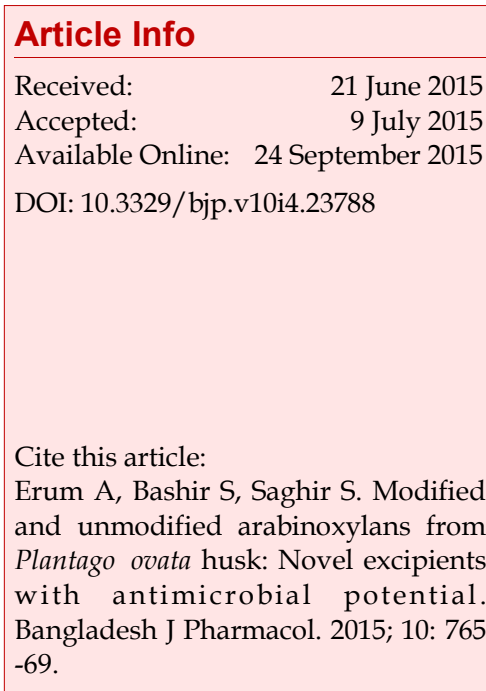

\begin{abstract}
Arabinoxylan is a polysaccharide of Plantago ovata, an indigenous plant of Pakistan and several European countries. In the present investigation arabinoxylan isolated from $P$. ovata husk was chemically modified i.e., carboxymethylated and was converted into its sodium salt. Physicochemical characterization of modified arabinoxylans was done. The antimicrobial assay of arabinoxylan and modified arabinoxylans was done by Kirby-Bauer disk diffusion method against Gram negative and Gram positive bacterial strains. Physicochemical properties of modified arabinoxylans were different from arabinoxylan yet were satisfactory for their use as pharmaceutical excipients. Arabinoxylan showed strong antimicrobial activity against Gram positive strains. However, it showed no antimicrobial activity against Gram negative strains. Carboxymethyl arabinoxylan showed maximum activity against $S$. epidermidis. It also showed activity against $S$. aureus and $P$. aeruginosa. Arabinoxylan $\mathrm{Na}$ showed concentration-dependent antimicrobial activity. This study provides the first report on the antibacterial properties of arabinoxylan and its modified forms.
\end{abstract}

\section{Introduction}

Among natural polymeric materials, polysaccharides and their derivatives are the most abundantly used in pharmaceutical formulations. Polysaccharides are considered to be most versatile polymers because they have structural diversity and create many linkages between monomer units. Chemical modification of polysaccharides results in improved process ability and generates industrially important derivatives (Lowman, 2000).

Plantago ovata is a hairy soft annual herb of 12-18 inches in height and belongs to the Plantaginaceae family. The polysaccharides extracted from the husk of $P$. ovata have been chemically characterized to contain a high proportion of hemicellulose which is the alkali soluble fraction of the husk. It consists of highly branched acidic arabinoxylan comprising of xylan backbone chain with xylose and arabinose forming the side chains
(Kennedy et al., 1979). Chemical modification of arabinoxylan can easily be performed because some xylp residues of the chain carry a single xylp moiety at position 2 with hydroxyl groups at position 2, 3, and 4 . The ethylation and carboxymethylation of arabinoxylan, isolated from P. ovata seed husk, were carried out (Saghir et al., 2008). Antimicrobial activity of ethanolic and methanolic extract of $P$. ovata has been reported against Gram positive and Gram negative bacteria (Motamedi et al., 2010).

In our previous studies, we evaluated arabinoxylan as potential pharmaceutical excipient (Sajid et al., 2014; Alia et al., 2014). During these studies, formulation containing arabinoxylan as excipient was not prone to microbial contamination and was assumed that it inhibits microbial growth. So, in present study attempt was made to evaluate antibacterial activity of arabinoxylan and its chemically modified forms. 


\section{Materials and Methods}

P. ovata husk was purchased from the local market in Pakistan. Other chemicals used were sodium hydroxide (Merck), ethanol (Riedel-de-Haen), sodium monochloroacetate (BDH Analar) and dimethylsulfoxide (Panreac).

\section{Isolation of arabinoxylan}

Arabinoxylan was isolated from the P. ovata husk by the method described elsewhere (Saghir et al., 2008) with slight modification. Percentage yield of gel was calculated by equation

Percentage $y i e l d=($ Weight of gel $/$ weight of husk $) \times 100$

Modification of arabinoxylan

Carboxymethylation

Arabinoxylan $(1.0 \mathrm{~g})$ was suspended in $72 \mathrm{~mL}$ ethanol. About $3.6 \mathrm{~mL}$ of $25 \%$ aqueous $\mathrm{NaOH}$ solution was added to the above reaction mixture and was vigorously stirred at room temperature. After 1 hour, sodium monochloroacetate $(2.64 \mathrm{~g})$ was added and the temperature of mixture was raised to $55^{\circ} \mathrm{C}$. The stirring of mixture was continued for 5 hours. The mixture was then filtered and suspended in $80 \%(\mathrm{v} / \mathrm{v})$ aqueous methanol. $\mathrm{pH}$ of solution was neutralized with dilute acetic acid and was filtered. The filtered product was washed with $20 \mathrm{~mL}$ ethanol five times and then dried for 2 days at $60^{\circ} \mathrm{C}$ (Sagir et al., 2008).

\section{Arabinoxylan $\mathrm{Na}$ salt formation}

Arabinoxylan $(1.0 \mathrm{~g}$ ) was dissolved in $50 \mathrm{~mL}$ of aqueous $\mathrm{NaOH}$ solution $(2.5 \%)$ to yield $2 \%$ solution. The solution was continuously stirred for 24 hours and was dried at $40^{\circ} \mathrm{C}$ in an oven under vacuum (James, 1939).

\section{Physicochemical characterization}

Physicochemical properties of modified arabinoxylans were determined such as their solubility, swelling index, tapped density, bulk density, angle of repose, compressibility index and $\mathrm{pH}$ (Sajid et al., 2014).

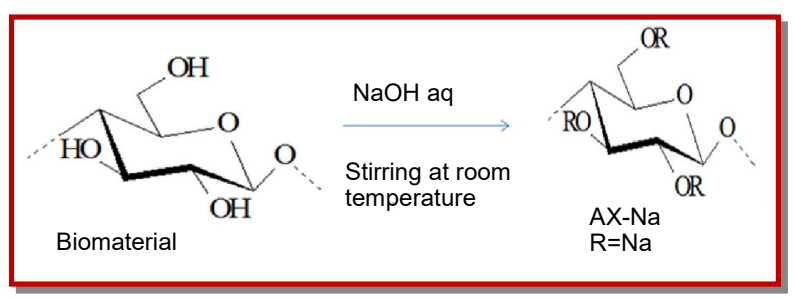

\section{Antimicrobial assay}

The antimicrobial assay of arabinoxylan, carboxymethyl arabinoxylan and arabinoxylan-Na against different Gram positive and Gram negative bacterial strains were conducted by Kirby-Bauer disk diffusion method.

Preparation of stock solution for antimicrobial assay

Stock solution $(5.0 \mathrm{~mL})$ of arabinoxylan $(5 \mathrm{mg} / \mathrm{mL})$ was prepared in DMSO. Arabinoxylan ( $25 \mathrm{mg}$ ) was added in $5 \mathrm{~mL}$ DMSO and stirred at $80^{\circ} \mathrm{C}$ for one hour until it was completely dissolved. Stock solutions of carboxymethyl arabinoxylan, A1 (5 mg/mL) and A2 (7.5 mg/ $\mathrm{mL})$ were prepared in distilled water. Arabinoxylan-Na stock solutions were made in $2.5 \% \mathrm{NaOH}$ containing (5 $\mathrm{mg} / \mathrm{mL}),(10 \mathrm{mg} / \mathrm{mL})$, and $20 \mathrm{mg} / \mathrm{mL}$ arabinoxylan-Na for solutions $\mathrm{A}, \mathrm{B}$ and $\mathrm{C}$ respectively.

\section{Microorganisms used}

The test organisms used were Gram positive strains such as Staphylococcus aureus (ATCC 25923), Actinomyces odontolyticus (ATCC 17929), Bacillus subtilis (ATCC 6633) and Staphylococcus epidermidis (ATCC 12228), and Gram negative strains such as Escherichia coli (ATCC 25922) and Pseudomonas aeruginosa (ATCC 27853).

\section{Kirby Bauer disc diffusion method}

About $0.1 \mathrm{~mL}$ of bacterial inoculums having turbidity adjusted according to McFarland's 1 as standard was spread uniformly on Mueller Hinton agar plates. Arabinoxylan, carboxymethyl arabinoxylan and arabinoxylanNa loaded $6 \mathrm{~mm}$ discs of Whatman No. 1 filter paper were placed on the surface of medium and plates were incubated for 24 hours at $37^{\circ} \mathrm{C}$. Ciprofloxacin loaded disc containing $0.1 \mu \mathrm{g} /$ disc was used as positive control while DMSO loaded disc was used as a negative control. In case of arabinoxylan-Na and carboxymethyl arabinoxylan, distilled water dipped disc was used as solvent control. At the end of incubation period, inhibition zones were measured in millimeters. These studies were performed in triplicate.

\section{Results and Discussion}

Arabinoxylan gel was successfully isolated from husk (Figure 1) and its yield was about $45 \%$ which was rated as good.

Modification of polysaccharide yield structurally and functionally altered derivatives having potential to be explored for their use in different fields. The polysaccharides which have undergone modification include starch (Chen et al., 2007), cellulose (Hon, 1996), chitosan (Rinaudo, 2006), psyllium (Singh and Sharma, 2010) and guar-gum (Dodi et al., 2011). Carboxymethylation of arabinoxylan with sodium monochloroacetate resulted in replacement of $\mathrm{OH}$ group with carboxymethyl group.

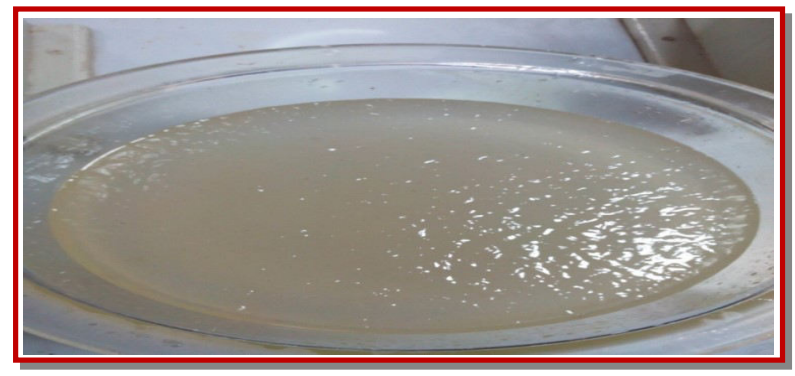

Figure 1: Extracted gel from Plantago ovata husk 
Most polysaccharides such as alginic acid, astragulus gummifer etc. are available in a variety of mono and multivalent cation salt form with varying properties (Abdul et al., 2014). In arabinoxylan- $\mathrm{Na}$, -OH group of arabinoxylan was replaced with sodium ion.

Carboxymethylation of arabinoxylan yielded water soluble derivative, which is in accordance with the literature as the solubility of gums is improved by carboxymethylation (Olusola et al., 2014). Carboxymethyl arabinoxylan was also soluble in $0.1 \mathrm{~N} \mathrm{HCl}$ and aqueous $\mathrm{NaOH}$. Arabinoxylan-Na was partially soluble in water having aqueous solubility of $42.8 \%$ (Table I). It was also soluble in $60 \%$ ethanol on slight heating and aqueous $\mathrm{NaOH}$ while it was insoluble in $0.1 \mathrm{~N} \mathrm{HCl}$ methanol and ethanol. Arabinoxylan-Na salt had reduced swelling than arabinoxylan with swelling index of $27 \pm 1 \%$ in distilled water which was due to presence of water soluble fraction within it. The moisture content of carboxymethyl arabinoxylan and arabinoxylan- $\mathrm{Na}$ was low as calculated from its loss on drying. The low moisture values suggest their suitability as excipient in formulations of moisture sensitive drugs. The knowledge of compressibility and flow indices is important for justifying the use of materials as excipients in a pharmaceutical formulation. So, according to values of bulk and tapped densities and their differences, compre -ssibility index was calculated (Table I). According to these parameters, carboxymethyl arabinoxylan was the freest flowing. Arabinoxylan-Na was also free flowing. The stability of most preparations is highly dependent on $\mathrm{pH}$. Excipient $\mathrm{pH}$ influences the $\mathrm{pH}$ of formulation. Therefore, $\mathrm{pH}$ of $1 \%$ solution of modified Arabinoxylans were determined by $\mathrm{pH}$ meter (Luiz et al., 2005). The $\mathrm{pH}$ of carboxymethyl arabinoxylan was 7.5 and for arabinoxylan-Na was 7.61 (Table I), which are almost neutral. This implies that they can be used as excipients in all types of drugs i.e. acidic, basic and neutral. These properties of modified arabinoxylans are different from arabinoxylan (Sajid et al., 2014).

Secondary metabolites of some plants showed antibacterial activity. The medicinal importance of phytochemical constituents has been established in the treatment of various diseases (Yadav and Munin, 2011). Antimicrobial activity of some polysaccharide gels such as gel from Durio zibethinus had been documented (Lipipun et al., 2002).

Arabinoxylan showed strong antimicrobial activity against Gram positive strains, arabinoxylan showed maximum activity against $B$. subtilis with zone of inhibition $35 \pm 2$ (Table II). It also showed activity against $S$. mutans with zone of inhibition $12 \pm 1.5, A$. odontolyticus with zone of inhibition $10 \pm 1$ and $S$. aureus with zone of inhibition $10 \pm 1$. However, it showed no antimicrobial activity against Gram negative strains $E$. coli and $P$. aeruginosa. This was most probably due to different cell membrane structure of Gram negative bacteria and presence of enzymes in periplasmic space that cause destruction of foreign particles (Bassam et al., 2004). Modification of arabinoxylan resulted in products carboxymethyl arabinoxylan and arabinoxylan- $\mathrm{Na}$ with altered structures and properties. Antimicrobial activity of modified arabinoxylans was quiet different from arabinoxylan. Etherification of arabinoxylan not only changed its structure, its physicochemical properties but also changed its antimicrobial spectra which was most probably because antimicrobial agents showed structure activity based relationship (George and Eleftherios, 2012).

In carboxymethyl arabinoxylan greater antimicrobial activity was shown by A1 than A2, which was most probably due to a very viscous solution that was unable

Table I

Physicochemical characterization of modified arabinoxylans

\begin{tabular}{|l|cc|}
\hline Parameters & Carboxymethyl arabinoxylan & Arabinoxylan-Na salt \\
\hline Solubility & $\begin{array}{l}\text { Soluble in water on slight heating and } \\
\text { in } 0.1 \mathrm{~N} \mathrm{HCl} \mathrm{solution,} \mathrm{aqueous} \mathrm{NaOH}, \\
\text { insoluble in ethanol and methanol }\end{array}$ & $\begin{array}{c}\text { Partially soluble in water } 42.8 \%, 60 \% \\
\text { ethanol on slight heating, } \\
\text { insoluble in } 0.1 \mathrm{~N} \mathrm{HCl} \text { and methanol }\end{array}$ \\
Swelling index in distilled water & - & $27 \pm 1$ \\
Loss on drying $(\%)$ & $0.4 \pm 0.0$ & $0.4 \pm 0.1$ \\
Bulk density $(\mathrm{g} / \mathrm{mL})$ & $0.5 \pm 0.0$ & $0.8 \pm 0.0$ \\
Tapped density $(\mathrm{g} / \mathrm{mL})$ & $0.6 \pm 0.0$ & $1.0 \pm 0.0$ \\
Compressibility index $(\%)$ & 14.3 & 18.2 \\
Angle of repose $\left({ }^{\circ}\right)$ & $29.0 \pm 1.1$ & $32.0 \pm 1.5$ \\
pH $(1 \%$ solution $)$ & $7.5 \pm 0.0$ & $7.6 \pm 0.0$ \\
\hline
\end{tabular}




\begin{tabular}{|c|c|c|c|c|c|c|c|}
\hline \multicolumn{8}{|c|}{ Table II } \\
\hline \multicolumn{8}{|c|}{ Antimicrobial susceptibility testing of modified arabinoxylans against bacterial strains } \\
\hline \multirow[t]{2}{*}{ Strains } & \multirow{2}{*}{$\begin{array}{c}\text { Positive control } \\
\text { ciprofloxacin } \\
(0.1 \mu \mathrm{g} / \text { disc })\end{array}$} & \multirow{2}{*}{$\begin{array}{c}\text { Arabinoxylan } \\
(\mu \mathrm{g} / \text { disc }) \\
100\end{array}$} & \multicolumn{2}{|c|}{$\begin{array}{l}\text { Carboxymethyl arabinoxylan } \\
\text { ( } \mu \mathrm{g} / \text { disc })\end{array}$} & \multicolumn{3}{|c|}{ Arabinoxylan-Na ( $\mu \mathrm{g} /$ disc $)$} \\
\hline & & & $\begin{array}{l}100 \\
\text { A1 }\end{array}$ & $\begin{array}{l}200 \\
\text { A2 }\end{array}$ & $\begin{array}{c}100 \\
\mathrm{~A}\end{array}$ & $\begin{array}{c}200 \\
\text { B }\end{array}$ & $\begin{array}{c}400 \\
C\end{array}$ \\
\hline \multicolumn{8}{|c|}{ Zone of inhibition (mm) } \\
\hline E. coli & 40 & 0 & 0 & 0 & 0 & $7 \pm 0$ & 0 \\
\hline S. aureus & 36 & $10 \pm 1$ & $21 \pm 2$ & $19 \pm 1.5$ & 0 & $17 \pm 2.5$ & $2 \pm 1.5$ \\
\hline B. subtilis & 40 & $35 \pm 2$ & 0 & $12 \pm 3$ & $3 \pm 0$ & $18 \pm 2$ & $12 \pm 1$ \\
\hline S. epidermidis & 34 & 0 & $26 \pm 2$ & $12 \pm 1$ & $5 \pm 1.5$ & $14 \pm 2$ & $3 \pm 1$ \\
\hline A. odontolyticus & 40 & $10 \pm 1$ & 0 & 0 & $3 \pm 1$ & $19 \pm 2$ & $8 \pm 2$ \\
\hline P. aeruginosa & 37 & 0 & $20 \pm 1$ & $20 \pm 2$ & $14 \pm 3$ & $19 \pm 1.5$ & $4 \pm 1.5$ \\
\hline S. mutans & 38 & $12 \pm 1.5$ & 0 & $10 \pm 1$ & $12 \pm 2$ & $15 \pm 1.4$ & $5 \pm 1$ \\
\hline
\end{tabular}

to penetrate the agar resulting in less zone of inhibition. Carboxymethyl arabinoxylan (A1) showed maximum activity against $S$. epidermidis with zone of inhibition $26 \pm 2$. It also showed activity against $S$. aureus with zone of inhibition $21 \pm 2$ as well as against Gram negative $P$. aeruginosa with zone of inhibition $20 \pm 1$. Carboxymethyl arabinoxylan (A2) was also effective against same strains as A1 but with less zone of inhibition (Table II). However, unlike A1 it was effective against $B$. subtilis with zone of inhibition $12 \pm 3$ and $S$. mutans with zone of inhibition $10 \pm 1$.

Similar to carboxymethyl arabinoxylan, arabinoxylan-Na also showed different antimicrobial spectrum from arabinoxylan. In arabinoxylan-Na antimicrobial activity was observed to be concentration dependent. Solution A $(100 \mu \mathrm{g} /$ disc $)$ showed maximum activity against $P$. aeruginosa with zone of inhibition $14 \pm 3$. It also showed activity against B. subtilis, S. epidermidis, A. odontolyticus, $P$. aeruginosa and S. mutans. Solution B (200 $\mu \mathrm{g} /$ disc $)$ showed more activity as compared to solution A i.e., greater zones of inhibition against $B$. subtilis, $S$. epidermidis, A. odontolyticus, P. aeruginosa and S. mutans (Table II). In addition, it also showed activity against $E$. coli and S. aureus. However, solution C (400 $\mu \mathrm{g} /$ disc), highest tested strength of arabinoxylan- $\mathrm{Na}$, showed least activity against all tested strains and no activity against E. coli. This was most probably due to highly concentrated gel that was unable to diffuse agar to inhibit bacterial growth.

\section{Conclusion}

Arabinoxylan, carboxymethyl arabinoxylan and arabinoxylan-Na possessed significant antibacterial activity. However, difference in intensity and spectrum of antimicrobial activity was due to variations in their structures. Maximum activity was shown by arabinoxylan-Na.

\section{References}

Abdul M, Nayyar P, Pramod KS. Novel polymers for mucoadhesive drug delivery. Int J Pharm. 2014; 4: 212-20.

Alia E, Sajid B, Shazia S, Sadia H, Amna B, Tahir M. Arabinoxylan isolated from ispaghula husk: A better alternative to commercially available gelling agents. Asian J Chem. 2014; 26: 8366-70.

Bassam AS, Ghaleb A, Dhaood AS, Naser J, Kamel A. Antibacterial activities of some plant extracts utilized in popular medicine in Palestine. Turk J Biol. 2004; 28: 99-102.

Chen L, Li X, Li L, Guo S. Acetylated starch-based biodegradable materials with potential biomedical applications as drug delivery systems. Curr Appl Phys. 2007; 7: 90-93.

Dodi G, Hritcu D, Popa MI. Carboxymethylation of guar gum: Synthesis and characterization. Cellulose Chem Technol. 2011; 45: 171-76.

George T, Eleftherios M. Antimicrobial drug discovery: Emerging strategies. UK, CPI group, 2012, pp 187-207.

Hon DNS. Chemical modification of cellulose. In: Polysaccharides in medicine and biotechnology. Severian D (ed). New York, Marcel Dekker, 1996.

James FW. Sodium lactate and method of manufacture. United States Patent Office, 2143362, 1939.

Kennedy JF, Sandhu JS, Southgate DAT. Structural data for the carbohydrate of ispaghula husk ex Plantago ovata Forsk. Carbohydr Res. 1979; 75: 265-74.

Lipipun V, Nantawanit N, Pongsamart S. Antimicrobial activity (in vitro) of polysaccharide gel from durian fruit-hulls. Songklanakarin J Sci Technol. 2002; 24: 31-38.

Lowman AM. Complexing polymers in drug delivery. In: Handbook of pharmaceutical controlled release technology. Wise DL (ed). New York, Marcel Dekkar, 2000.

Luiz ALS, George GO, Pedro RP, Peter CS. Dry granulation and compression of spray-dried plant extracts. AAPS Pharm Sci. 2005; 6: 359-66. 
Motamedi H, Darabpour E, Gholipour M, Nejad SMS. Antibacterial effect of ethanolic and methanolic extracts of Plantago ovata and Oliveria decumbens endemic in Iran against some pathogenic bacteria. Int J Pharmacol. 2010; 6: 117-22.

Olusola A, Toluwalope GA, Olutayo O. Carboxymethylation of Anacardium occidentale L. rxudate gum: Synthesis and characterization. Sch Acad J Pharm. 2014; 3: 213-16.

Rinaudo M. Chitin and chitosan: Properties and applications. Prog Polym Sci. 2006; 31: 603-32.

Saghir S, Iqbal M S, Hussain M A, Koschella A, Heinze TH. Structure characterization and carboxymethylation of arabi- noxylan isolated from ispaghula (Plantago ovata) seed husk. Carbohydr Polym. 2008; 74: 309-17.

Sajid B, Alia E, Shazia S, Umme Ruqia T, Ayesha R. Physicochemical characterization and evaluation of suspending properties of arabinoxylan from ispaghula (Plantago ovata) husk. Pak J Pharm Sci. 2014; 27: 1761-66.

Singh V, Sharma V. Design of psyllium-PVA-acrylic acid based novel hydrogel for use in antibiotic drug delivery. Int J Pharm. 2010; 389: 94-106.

Yaday RNS, Munin A. Phytochemical analysis of some medicinal plants. J Phytol. 2011; 3: 10-14.

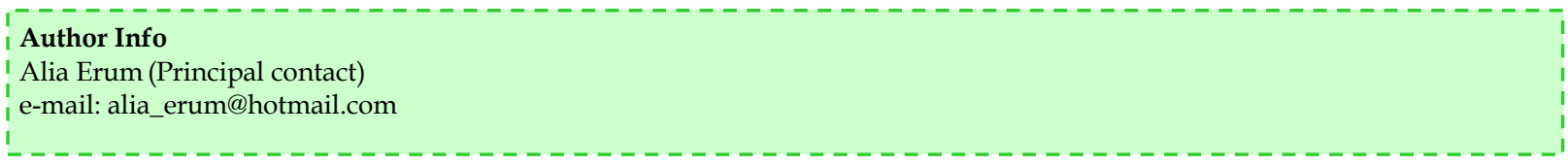

